\title{
The Use of Bigrams to Enhance
}

\section{Text Categorization}

Chade-Meng Tan*

Department of Computer Science

University of California

Santa Barbara, CA 93106

Tel: (805)893-3866

FAX: (805)893-8553

meng@google.com
Yuan-Fang Wang** Communication Engineering

Taejon University

Taejon, 300-716, KOREA

Tel: 82(42)280-2551

FAX: 82(42)284-0109

cdlee@dragon.taejon.ac.kr

* Current Address: Google Inc., 2400 Bayshore Pkwy, Mountain View, CA 94043.

Tel: (650)330-0100 x 1146; FAX: (650)618-1499

** Corresponding author 


\section{Abstract}

In this paper, we present an efficient text categorization algorithm that generates bigrams selectively by looking for ones that have an especially good chance of being useful. The algorithm uses the information gain metric, combined with various frequency thresholds. The bigrams, along with unigrams, are then given as features to a Naï ve Bayes classifier. The experimental results suggest that the bigrams can substantially raise the quality of feature sets, showing increases in the break-even points and F1 measures. The McNemar test shows that in most categories the increases are very significant. Upon close examination of the algorithm, we conclude that the algorithm is most successful in correctly classifying more positive documents, but may cause more negative documents to be classified incorrectly.

Keywords: information retrieval, text categorization, text classification, machines learning. 


\section{Introduction}

Text categorization (also known as text classification) is, quite simply, the automated assignment of natural language texts to predefined categories based on their content (see Sebastiani, 2000, for an introduction). Its applications include indexing texts to support document retrieval, extracting data from texts, and aiding humans in these tasks (Lewis 1992b). The performance of standard text categorization techniques on standard test corpora has been quite encouraging. Apté et al. (1998), for example, reported an 87.8\% precision/recall breakeven point for the Reuters- 21578 corpus.

At present, text categorization techniques are predominantly keyword-based. Many researchers in the field have used different classifiers, but most of them treat a document as a bag of words (BOW), that is, identify terms with all the words occurring in the document, and perform categorizations based mainly on the presence or absence of keywords. Billsus and Pazzani (1997) showed that various categorization techniques produced similar results for BOWbased features ${ }^{1}$. Yang and Pederson (1997) and Lewis and Ringuette (1994) came to the similar conclusions.

${ }^{1}$ In categorization features are often used to refer to the same kind of entity as terms in information retrieval. In this paper, we use them interchangeably as appropriate. 
In a number of experiments (Lewis, 1992a, 1992b, 1992c; Apté et al., 1994), it was found that the use of phrases ${ }^{2}$ actually caused text categorization performance to degrade. Despite of these discouraging results, investigations of using phrases have been actively pursued (Mladeni(ग) and Grobelnik 1998; Fürnkranz, 1998; Schütze et al., 1995; Schapire et al., 1998).

This paper presents our attempt to improve categorization performance by automatically extracting and using phrases, especially two-word phrases (hereafter bigrams). Our algorithm generates high quality bigrams by using the information gain (infogain) metric, combined by various frequency thresholds. The experimental results suggest that the bigrams can substantially raise the quality of feature sets, showing increases in the break-even points and F1 measures.

The remainder of the paper is organized as follows: Section 2 reviews related works and justifies our study, Section 3 discusses our algorithm and its implementation, Section 4 presents our experimental results, Section 5 analyzes the results and the performance of the algorithm, and Section 6 suggests further work.

\footnotetext{
${ }^{2} \mathrm{~A}$ phrase refers to "an indexing term that corresponds to the presence of two or more single word indexing terms" (Lewis, 1992a, p.35), whether the notion of phrase is motivated syntactically or statistically.
} 


\section{The Use of Phrases in Text Categorization}

Lewis (1992a, 1992b, 1992c) extensively examined the use of phrases in text categorization. He performed part-of-speech parsing on the text and used all noun phrases that occurred at least twice as features. He showed that phrases gave worse performance than single words. The degradation in performance was due to that high dimensionality, low frequency, and high degree of synonymy using phrases as features outweigh the advantages phrases had in lowering ambiguity (Lewis 1992b). Lewis (1992a) gave a more detailed analysis of why phrases failed to do well. He stated 6 characteristics that were desirable for features for text categorization:

1. Small number of indexing terms

2. Flat distribution of values for an indexing term

3. Lack of redundancy among terms

4. Low noise in indexing term values

5. Lack of ambiguity for linguistically derived terms

6. Terms should be related to the classes to be induced

Phrases tend to do well on the criterion 5, but badly on the first 4 criteria. First, the number of indexing terms for phrases may be large. If there are $d$ words, for example, there are potentially $d^{2}$ phrases. Second, most of these $d^{2}$ phrases are likely to take on the value of 0 for all documents, while the rest might be 0 for most documents, implying that the distribution of the indexing terms may be far from flat. Third, phrases are likely to have higher redundancy caused 
by high synonymy: if each word in a phrase has $k$ synonyms, then $k^{2}$ phrases could have the same meaning. Lastly, phrases tend to be noisier. If two phrases have an identical meaning, we would want them to be assigned to the same documents. However, in most natural language texts, the opposite is the case. In short texts, there are simply not enough references to a concept for all the synonymous phrases to be used. Having more phrases with the same meaning only worsens the problem.

Several efforts have been made to circumvent the possible problems posed by using phrases and some research results showed that the addition of $n$-grams (sequences of words of length $n$ ) to the BOW representation indeed improved performance. However, sequences of length $n>3$ were shown to be not useful and might decrease the performance.

Mladeni(D) and Grobelnik (1998) generated new features based on word sequences of different length up to 5. Learning was performed using NB classifier on feature- vectors, where only highly scored features according to term frequency were used. The performance was evaluated using Yahoo text hierarchy. They showed that using word sequences of length up to 3 instead of using only single words improved the performance, while longer sequences in average had no influence on the performance.

Fürnkranz (1998) came to a similar conclusion. He used an algorithm based on the APRIORI algorithm for efficiently generating features using term frequency and document frequency as criteria. His experimental results indicated that word sequences of length 2 or 3 were most useful, while using longer sequences reduced categorization performance. He also 
showed that moderate frequency-based pruning of the feature set could be useful, while heavy frequency-based pruning resulted in a performance decrease, thus contradicting the findings by Yang and Pederson (1997) and Lewis (1992b) that heavy pruning may improve performance.

Schütze et al. (1995) applied dimensionality reduction techniques to overcome computational intensity and overfitting in solving document routing, a problem of statistical text categorization. As terms they used single words and two-word phrases that were chosen by term frequency as an evaluation measure. Their findings showed that a reduced feature space was both practical and beneficial for document routing.

Schapire et al. (1998) used words and phrases in applying Rocchio's algorithm for text filtering. They also used term frequency as a criterion to choose which phrases to select. However, they were not clear as to what a phrase meant.

Our approach is different in many aspects from the above-mentioned studies. First, we use bigrams in addition to, not in place of, single words. Second, we are highly selective of the bigrams we use. The number of bigrams our algorithm finds is no more than $2 \%$ of the number of single words as we try to avoid the problem of high dimensionality. With continued use of single words in combination with a small number of added bigrams, our approach fares only slightly worse for criteria $1-4$ mentioned in Lewis (1992a). Finally, we use infogain in addition to document frequency and term frequency to choose which bigrams to use. This means that the bigrams that we select are likely to be good discriminators and less likely to be noisy. 


\section{Our Algorithm and Its Implementation}

The idea of our algorithm is simply to find bigrams in which at least one of the constituent words (hereafter unigrams) has a minimum document frequency in at least one of the categories. Intuitively, what our algorithm does is to first find the list of unigrams that appear in a significant number of documents, and use them as seeds. All the training documents are then scanned and we gather all bigrams where at least one of its component unigrams is a seed. We then select only the bigrams, among those extracted, with high occurrences and infogain. Figure 1 shows the pseudo-code of our algorithm.

1. Find $\mathrm{S}=\left\{\right.$ set of words that occur in at least $d f_{-}$seed $*$ number of documents in at least 1 category $\}$

2. Set $\mathrm{B}=$.

3. For each document in the training set

4. \{

5. Preprocess document by removing all numbers, punctuation and words in a stoplist.

6. For each pair of adjacent words (w1, w2) in preprocessed document

7. If (w1 is in S or w2 is in S), add bigram "w1+w2" to B.

8. $\quad$ \}

9. For each $\mathrm{b}$ in $\mathrm{B}$

10. \{

11. For each category c

12. \{

13. If (b occurs in less than $d f$ bigram * number of documents in category c)

14. OR (b occurs in fewer than tf_bigram times in all documents)

15. remove $b$ from $B$

16. $\}$

17. If ( $\mathrm{b}$ is not removed and $\mathrm{b}$ has infogain $<$ ig_bigram) remove $\mathrm{b}$ from $\mathrm{B}$

18. $\}$

19. Output B

Figure 1. Bigram extraction algorithm 
We performed some pilot experiments and the number 0.01 seemed to be good for $d f \_$seed and 0.005 for $d f \_$bigram. For ig_bigram, we set it to the infogain of the single word at position igat_unigram in the list of unigrams sorted by decreasing infogain. igat_unigram was set at approximately 1 percent of all unigrams. We also set max_bigram at 1.5 times igat_unigram. In our experiments, igat_unigram was set to 1500 for the Yahoo! Science corpus (corresponding roughly to $1 \%$ of the $160,000+$ unique unigrams) and for the Reuters corpus, it was set to 400 (about $1 \%$ of the $42,000+$ unique unigrams). Finally, we set $t f \_$bigram to 3.

Note that the algorithm preprocesses the training set to collect the occurrence statistics of each unigram and to calculate its infogain.

Our programs were written in $\mathrm{C}$ and built upon Andrew McCallum's Bag of Words Library (libbow) (McCallum 1996). The libbow distribution ships with a text categorization front-end known as rainbow. We made use of rainbow for building models of our data and for its built-in NB classifier. Stemming was not used in categorization.

\section{Experiments}

\subsection{Test Corpora}

Two corpora were used for the experiments. One was a collection of webpages pointed to by the Yahoo! Science hierarchy gathered in July 1977 (McCallum, personal communication), 
which we call the "Yahoo-Science" corpus. The other was the Reuters-21578 corpus, one of the standard benchmarks for text categorization tasks.

All documents in both corpora were preprocessed to retain only the bodies of each document by discarding headers and the likes. In addition, all numbers and punctuation were removed and all words set to lower case. Finally, all stopwords were removed using a standard stopwords list (Salton and McGill, 1983).

\subsubsection{Yahoo-Science}

The collection originally contained 14,869 documents in 30 top-level categories. For each top-level category, "flattening" was performed: we moved all documents in its subcategories to the top-level category and removed all the sub-categories. We decided to concentrate our attention on the 10 largest categories. All other categories were deleted and their documents moved to the "Others" category. We also added 3,410 documents gathered from the Yahoo! Health hierarchy to the "Others" category. The idea was to make our categorization experiments a little more realistic by adding a significant number of totally unrelated documents.

In the end, we had 14,477 unique documents in our collection (with some documents existing in more than 1 category). These documents contained a total of 160,975 unique words.

\subsubsection{Reuters -21578}

This collection contained 22,173 unique documents with 42,418 unique words. Since out of 135 categories 14 had no documents and many others had very few documents (53 categories 
had 10 or fewer documents, and 97 less than 100 documents), we decided to concentrate our attention on the 12 largest categories.

\subsection{Evaluation Measures}

The standard performance measures for text categorization are recall and precision (Apté et al. 1994). Each level of recall is associated with a level of precision. In general, the higher the recall, the lower the precision, and vice versa. The point at which recall equals precision is the break-even point $(B E P)$, which is often used as a single summarizing measure for comparing results.

There are instances where a real BEP does not exist. Other useful measures for evaluating the effectiveness of classifiers are the F measures (Yang and Liu 1999). Among many variants of them, F1 measure is used in this study.

These scores are often computed for the binary decisions on each individual category first and then averaged. Both macro-averaging and micro-averaging are used in this study.

\subsection{Experimental Results on Yahoo-Science Corpus}

\subsubsection{Experimental Tasks}

The categorization task was treated as a series of sub-tasks, each performing binary categorization on the chosen category except for the "Other". For each category, we classified whether a document is in the category (a "Is" document) or not in the category (a "Not" 
document). To minimize the variations due to the selection of training data, we used a "3-fold cross-validation": for each category, we partitioned the data into three sets, with "Is" and "Not" documents equally distributed among them. We then ran three experiments for each category, each experiment used two of the three sets as training set and the remaining as the test set and calculated the average of the three runs as the final result for the category. These experiments were run for categorization using only unigrams, and also for categorization using bigrams as well as unigrams.

\subsubsection{Bigrams extracted}

The algorithm found bigrams effectively. It found average of 2520 unique bigrams over 10 categories and 1426 bigrams on each run (see Tan, 2000, for more detailed experimental results).

Table 1 shows some of the 4439 unique bigrams found by our algorithm for the Computer Science category. The algorithm was very successful at extracting bigrams that accurately describe some concepts, such as "computer+science", "genetic+algorithm", "source+code", and "end+user". Yet, there were bigrams found from the negative category, such as "health+care". Other categories showed similar findings. 
Table 1. Sample bigrams extracted for Computer Science Category

\begin{tabular}{|c|c|c|}
\hline $\begin{array}{l}\text { computer+science } \\
\text { artificial+intelligence } \\
\text { neural+networks } \\
\text { department+computer } \\
\text { technical+reports } \\
\text { machine+learning } \\
\text { natural+language } \\
\text { neural+network } \\
\text { computer+vision } \\
\text { human+computer } \\
\text { genetic+algorithms }\end{array}$ & $\begin{array}{c}\text { Evolutionary+computation } \\
\text { school+computer } \\
\text { information+technology } \\
\text { time+systems } \\
\text { research+artificial } \\
\text { electrical+engineering } \\
\text { papers+technical } \\
\text { source+code } \\
\text { vision+robotics } \\
\text { intelligent+agents } \\
\text { higher+order }\end{array}$ & $\begin{array}{c}\text { systems+fuzzy } \\
\text { joint+conference } \\
\text { association+computational } \\
\text { robotics+institute } \\
\text { finite+state } \\
\text { ai+related } \\
\text { speech+technology } \\
\text { message+passing } \\
\text { end+user } \\
\text { iee+transactions } \\
\text { health+care }\end{array}$ \\
\hline
\end{tabular}

Our observation was that the use of bigrams increased the infogain, thus improving the quality of the features. On average in our experiment, 35.2 out of the top 100 features (in terms of infogain) were bigrams. This is pretty good, considering that there was only an average of 1,426 bigrams and more than 160,000 unigrams in our experiment. Another observation we made was that the bigrams improved the overall quality of the feature set. Without bigrams, the average infogain of all the categories was 3.88963e-05. When the bigrams were added, it increased by $31 \%$ to $5.09621 \mathrm{e}-05$. Many of the bigrams were of significantly higher quality than its constituent words. For example, "computer+science" was better than "computer" or "science."

\subsubsection{Impact on performance}

Figure 2 shows the Precision-Recall graph that summarizes the overall categorization results for Yahoo-Science. As the figure shows, the overall performance improved when both unigrams and bigrams were used. 


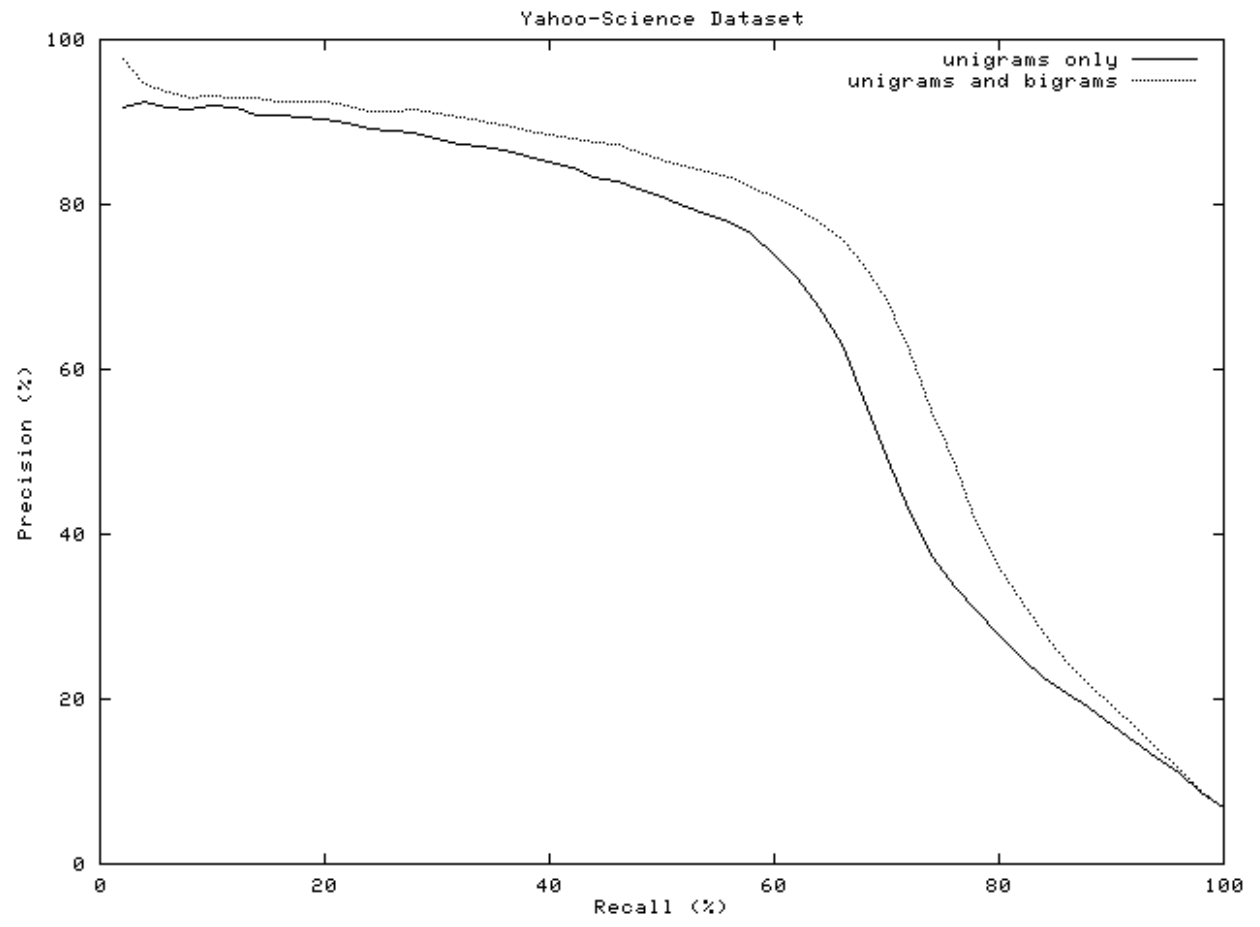

Figure 2. Precision-Recall graph for Yahoo-Science

Table 2 summarizes the results for the entire corpus. Both BEP and F1 measure showed that the performance of the classifier improved on each category when both unigrams and bigrams were used. Five of the ten categories showed more than $10 \%$ increases, with the highest at $27.6 \%$ in BEP and $28.6 \%$ in $\mathrm{F} 1$ measure. 
Table 2. Performance improvements on Yahoo-Science

\begin{tabular}{|c|c|c|c||c|c|c|}
\hline Category & $\begin{array}{c}\text { BEP } \\
\text { (w/o bigrams) }\end{array}$ & $\begin{array}{c}\text { BEP } \\
\text { (with bigrams) }\end{array}$ & Improvement & $\begin{array}{c}\text { F1 Measure } \\
\text { (w/o bigrams) }\end{array}$ & $\begin{array}{c}\text { F1 Measure } \\
\text { (with bigrams) }\end{array}$ & Improvement \\
\hline agriculture & 0.4295 & 0.5092 & $18.6 \%$ & 0.453 & 0.540 & $19.2 \%$ \\
\hline astronomy & 0.6950 & 0.7532 & $8.4 \%$ & 0.688 & 0.752 & $9.3 \%$ \\
\hline biology & 0.5256 & 0.5788 & $10.1 \%$ & 0.519 & 0.599 & $15.4 \%$ \\
\hline computer science & 0.4383 & 0.5591 & $27.6 \%$ & 0.458 & 0.589 & $28.6 \%$ \\
\hline earth sciences & 0.6229 & 0.6844 & $9.9 \%$ & 0.635 & 0.689 & $8.5 \%$ \\
\hline engineering & 0.6947 & 0.7183 & $3.4 \%$ & 0.693 & 0.720 & $3.9 \%$ \\
\hline mathematics & 0.4701 & 0.5628 & $19.7 \%$ & 0.497 & 0.595 & $19.7 \%$ \\
\hline physics & 0.5209 & 0.6077 & $16.7 \%$ & 0.529 & 0.600 & $13.4 \%$ \\
\hline space science & 0.5397 & 0.5619 & $4.1 \%$ & 0.621 & 0.636 & $2.4 \%$ \\
\hline zoology & 0.8489 & 0.8531 & $0.5 \%$ & 0.839 & 0.841 & $0.2 \%$ \\
\hline $\begin{array}{c}\text { Overall } \\
\text { (micro-average) }\end{array}$ & 0.6509 & 0.6954 & $6.8 \%$ & 0.661 & 0.705 & $6.7 \%$ \\
\hline $\begin{array}{c}\text { Average } \\
\text { (macro-average) }\end{array}$ & 0.57856 & 0.63885 & $11.9 \%$ & 0.5932 & 0.6561 & $12.1 \%$ \\
\hline
\end{tabular}

\subsection{Experimental Results on Reuters-21578}

\subsubsection{Experimental Tasks}

For this experiment, we used the "ModLewis" raining/test split (13625/6188, with 1765 unused). Since a standard split already exists, we did not perform the resource-consuming validation test.

\subsubsection{Bigrams extracted}

Like the Yahoo-Science experiment, the algorithm was very successful at extracting bigrams that accurately describe some concepts. Examples included "federal+reserve", "united+states", "soviet+union", "central+bank", and "crude+oil". However, once again, there 
were bigrams that were captured which did not necessarily describe the category. For example, "washington+march" in grain was formed because many of the articles on the topic were filed from Washington in March.

On average, 44.6 out of the top 100 features (in terms of infogain) were bigrams, which was even better than the case for Yahoo-Science. There was only an average of 531 bigrams and about 40,000 unigrams. Again, we observed that the bigrams improved the overall quality of the feature set. Without bigrams, the average infogain of the features over all the categories was 4.42648e- 05 . When the bigrams were added, it increased by $45.8 \%$ to $6.45557 \mathrm{e}-05$.

\subsubsection{Impact on performance}

As Figure 3 shows, the overall performance improved when both unigrams and bigrams were used. 


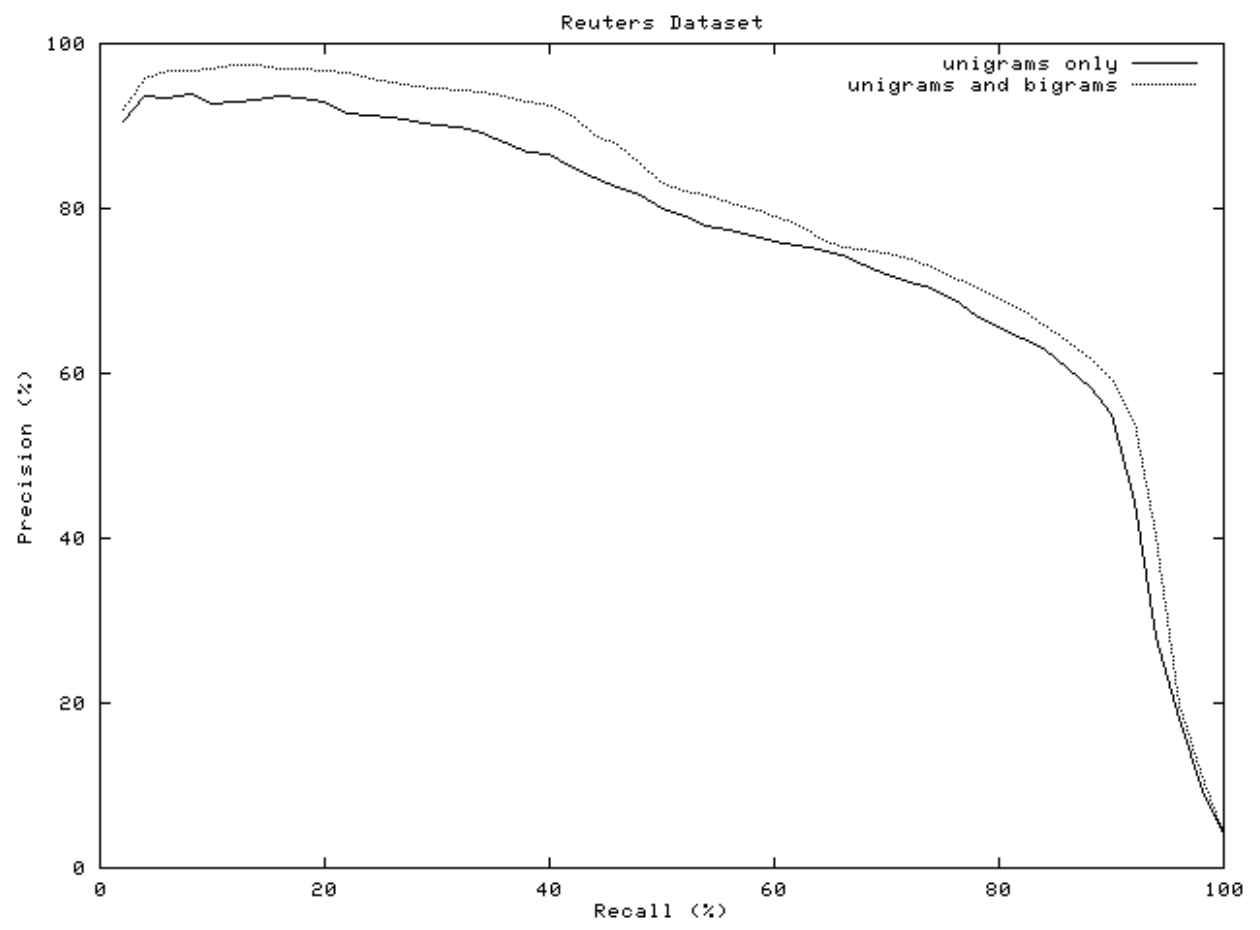

Figure 3. Precision-Recall graph for Reuters-21578

Table 3 summarizes the results for the entire corpus. BEP increased in all categories, with the highest at $21.4 \%$. However, the performance as measured by F1 was mixed. While the largest improvement remained at $27.1 \%$, five out of twelve categories showed a drop. The possible reasons of the degradation will be examined later. 
Table 3. Performance improvements on Reuters-21578

\begin{tabular}{|c|c|c|c||c|c|c|}
\hline Category & $\begin{array}{c}\text { BEP } \\
\text { (w/o bigrams) }\end{array}$ & $\begin{array}{c}\text { BEP } \\
\text { (with bigrams) }\end{array}$ & Improvement & $\begin{array}{c}\text { F1 Measure } \\
\text { (w/o bigrams) }\end{array}$ & $\begin{array}{c}\text { F1 Measure } \\
\text { (with bigrams) }\end{array}$ & Improvement \\
\hline acq & 0.7269 & 0.7325 & $0.8 \%$ & 0.680 & 0.669 & $-1.6 \%$ \\
\hline corn & 0.4956 & 0.6018 & $21.4 \%$ & 0.503 & 0.500 & $-0.6 \%$ \\
\hline crude & 0.7599 & 0.7968 & $4.8 \%$ & 0.790 & 0.796 & $0.8 \%$ \\
\hline $\mathrm{dlr}$ & 0.5169 & 0.5619 & $8.7 \%$ & 0.457 & 0.581 & $27.1 \%$ \\
\hline earn & 0.8177 & 0.8379 & $2.5 \%$ & 0.765 & 0.787 & $2.9 \%$ \\
\hline grain & 0.7291 & 0.7826 & $7.3 \%$ & 0.710 & 0.703 & $-1.0 \%$ \\
\hline interest & 0.6517 & 0.6966 & $6.9 \%$ & 0.664 & 0.678 & $2.1 \%$ \\
\hline money-fx & 0.6205 & 0.6427 & $3.6 \%$ & 0.661 & 0.656 & $-0.8 \%$ \\
\hline oilseed & 0.4211 & 0.4843 & $15.0 \%$ & 0.395 & 0.462 & $17.0 \%$ \\
\hline ship & 0.6816 & 0.6928 & $1.6 \%$ & 0.681 & 0.695 & $2.1 \%$ \\
\hline trade & 0.4766 & 0.5192 & $8.9 \%$ & 0.515 & 0.550 & $6.8 \%$ \\
\hline wheat & 0.6714 & 0.6993 & $4.2 \%$ & 0.624 & 0.591 & $-5.3 \%$ \\
\hline $\begin{array}{c}\text { Overall } \\
\text { (micro-average) }\end{array}$ & 0.7146 & 0.7334 & $2.6 \%$ & 0.700 & 0.705 & $0.7 \%$ \\
\hline $\begin{array}{c}\text { Average } \\
\text { (macro-average) }\end{array}$ & 0.6308 & 0.6707 & $6.3 \%$ & 0.620 & 0.639 & $3.0 \%$ \\
\hline
\end{tabular}

\subsection{McNemar's Test}

So far we have shown that the algorithm enhanced text categorization in most categories. However, how significant were the improvements? Was the better performance of the classifier really due to the addition of bigrams? Could we safely say that our algorithm attributed to the improvements? To answer these questions we ran a statistical test.

\subsubsection{Procedure}

McNemar's test (Dietterich, 1997) is a statistical process that can validate the significance of differences between two classifiers. Let $\hat{f}_{A}$ be the classifier trained with 
unigrams only and $\hat{f}_{B}$ be the one with both unigrams and bigrams. We tested them and recorded how each category was classified to construct the following contingency table:

\begin{tabular}{|c|c|c|}
\hline Number of Categories & Correct by $\hat{f}_{A}$ & Incorrect by $\hat{f}_{A}$ \\
\hline Correct by $\hat{f}_{B}$ & $n_{00}$ & $n_{01}$ \\
\hline Incorrect by $\hat{f}_{B}$ & $n_{10}$ & $n_{11}$ \\
\hline
\end{tabular}

The McNemar's test is based on the idea that there is little information in the numbers of categories for which both classifiers get the correct results, or for which both get an incorrect results; it is based entirely on the values of $n_{01}$ and $n_{10}$. Under the null hypothesis $\left(H_{0}\right)$, the two algorithms should have the same error rate, meaning $n_{01}=n_{10}$. It is a $\chi^{2}$ test in disguise and performs a test using the following statistic:

$$
\frac{\left(\left|n_{01}-n_{10}\right|-1\right)^{2}}{n_{01}+n_{10}} \text {. }
$$

If the $H_{0}$ is correct, then the probability that this number is bigger than $\chi_{1,0.95}^{2}=3.841459$ is less than 0.05 . So we may reject the $H_{0}$ in favor of the hypothesis that the two algorithms have different performance. 


\subsubsection{Test Results}

Table 4 shows the results of performing McNemar's test on all categories. Overall, the test exhibited that the improvements made by employing bigrams were really due to the algorithm the current study proposed. Yet, as shown in the table, some categories exhibited very high degree of significance between the two algorithms, while a few categories, especially in Reuter-21578, fell below the significant level.

Table 4. Results of McNemar's Test

\begin{tabular}{|c|c|c|c|c|c|}
\hline \multirow{14}{*}{ 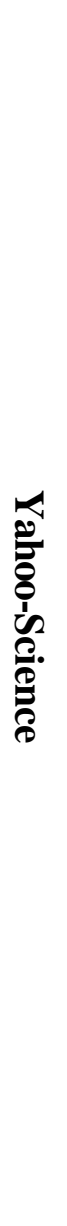 } & Category & p-value & \multirow{14}{*}{ 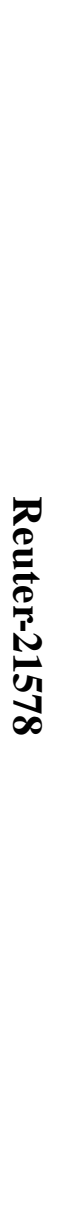 } & Category & p-value \\
\hline & agriculture & $4.902 \mathrm{e}-10$ & & acq & $4.77 \mathrm{e}-06$ \\
\hline & astronomy & $9.326 \mathrm{e}-15$ & & corn & 0.01192 \\
\hline & biology & $6.98 \mathrm{e}-10$ & & crude & 1.0 \\
\hline & computer science & $2.666 \mathrm{e}-11$ & & $\mathrm{dll}$ & 1.0 \\
\hline & earth sciences & $2.516 \mathrm{e}-09$ & & earn & $2.065 \mathrm{e}-14$ \\
\hline & engineering & $2.277 \mathrm{e}-08$ & & grain & 0.332 \\
\hline & mathematics & $5.679 \mathrm{e}-13$ & & interest & 0.2561 \\
\hline & physics & 0.001671 & & money-fx & 0.5959 \\
\hline & space science & 0.8445 & & oilseed & 0.5403 \\
\hline & zoology & 0.1360 & & ship & 0.4497 \\
\hline & & & & trade & 0.4881 \\
\hline & & & & wheat & 0.002346 \\
\hline & Overall & $2.2 \mathrm{e}-16$ & & Overall & $1.643 \mathrm{e}-14$ \\
\hline
\end{tabular}




\section{Discussion}

\subsection{Summary of the results}

The results of our experiments were very encouraging. Contrary to many research findings that the use of multi-word phrases caused the performance of text categorization to drop, our experiments showed that our approach to the extraction and use of bigrams could significantly enhance the performance. In all categories the BEP increased, with the highest at 27.6\%. The performance measured by the F1, however, was a little more mixed. While the figures showed improvements in all categories in Yahoo-Science, five out of the twelve categories actually showed a drop in Reuters-21578. The results of McNemar's test indicated that the improvements made by bigrams were really due to a better algorithm. Nonetheless, some categories exhibited a very high degree of significance, while a few categories fell below the significant level.

Even though we were able to show overall improvements using both BEP and F1 measure and McNemar test supported the improvements were significant, the uneven numbers raised some issues. We will discuss them in some detail. 


\subsection{Low Performance Increases in Some Categories}

It is immediately apparent that some categories benefited much more from the addition of bigrams than others. Why is it, then, that some categories did not do significantly better with bigrams?

\subsubsection{Adequacy of Unigrams}

There were cases where unigrams were adequate in describing the concepts in a category. For illustration, examine Table 5, which shows the top 10 terms in the Computer Science and Zoology categories.

Table 5. Top 10 terms in Computer Science and Zoology (numbers show infogain)

\begin{tabular}{|c|c|}
\hline Computer Science & Zoology \\
\hline 1.643893e-02 computer+science & $4.979735 \mathrm{e}-02 \quad \operatorname{dog}$ \\
\hline $1.596046 \mathrm{e}-02$ artificial+intelligence & $4.132053 \mathrm{e}-02$ pet \\
\hline $1.525400 \mathrm{e}-02$ cs & $3.748218 \mathrm{e}-02$ dogs \\
\hline $1.447255 \mathrm{e}-02$ computer & $3.436644 \mathrm{e}-02$ cat \\
\hline $1.327625 \mathrm{e}-02 \mathrm{acm}$ & $3.189234 \mathrm{e}-02$ breed \\
\hline $1.217493 \mathrm{e}-02$ intelligence & $3.059738 \mathrm{e}-02$ cats \\
\hline 1.196061e-02 artificial & $2.832415 \mathrm{e}-02$ pets \\
\hline $1.076363 \mathrm{e}-02$ neural & $2.695699 \mathrm{e}-02$ animals \\
\hline $9.479605 \mathrm{e}-03 \quad$ systems & $2.237056 \mathrm{e}-02$ animal \\
\hline $9.369088 \mathrm{e}-03$ ai & $2.224228 \mathrm{e}-02$ university \\
\hline
\end{tabular}

We see that single terms could describe the concepts in Zoology adequately. While the top two terms in Computer Science ("computer+science", "artificial+intelligence") could not have been adequately described by single words, all of the tops ten terms in Zoology were 
unigrams that described concepts very well in this collection. In fact, unigrams were so strong in this category that only 5 bigrams made it to the list of 100 top terms (as opposed to the average of 33.2 for all categories).

Given the strength of the unigrams in such cases, the addition of bigrams did not make much of a difference for this particular category.

\subsubsection{Over-Emphasis of Common Concepts}

Some categories in Reuters-21578 showed decreases in the F1 measure when bigrams were added. The major cause of it was that some bigrams over-emphasized concepts that were common to documents in both positive and negative categories.

Here is an example. In the acq category of Reuters-21578, the addition of bigrams caused recall to increase from 0.927 to 0.935 but caused precision to drop from 0.537 to 0.521. That drop caused the F1 measure to go down. The immediate reason for the drop in the precision was the increased number of false positives. Figure 4 shows an example of one such case.

MCF FINANCIAL PLANS INITIAL PUBLIC OFFERING ENCINO, Calif., April 8 - MCF financial Corp said it filed a registration statement with the Securities and Exchange Commission covering a proposed initial public offering of 1,075,000 common shares.

MCF said proceeds from the offering will be used to repay debt, to purchase loan participation interests and for working capital.

MCF is engaged in the commercial finance business.

\section{Figure 4. Case of a false positive caused by addition of bigrams}


Even though this document was originally classified as "NOT acq", it was changed to $a c q$, since it contained the bigrams which were more common in the acq category than the "NOT acq" category, such as "common+shares", "financial+corp", and "securities+exchange". The false positives occurred because concepts that were common to many documents in both positive and negative category were over-emphasized.

We discovered that this problem occurred much more often in Reuters-21578. It is not clear at this time why this was the case. One likely cause could be that Reuters-21578 started at a much higher recall rate than Yahoo-Science, hence it was more vulnerable to false positives. We also speculated that one possible cause could be that the documents in Reuters21578 referred to fewer concepts on average than Yahoo-Science. On average, there were 1.91 unique unigrams per document in Reuters-21578, while 11.1 unique unigrams per document in Yahoo-Science. Finally, the problem might be solved if our algorithm could find the right bigrams to reinforce the negative category. For example, if we had found bigrams such as "public+offering" and "repay+debt" to reinforce the "Not $a c q$ " category, then documents such as the one in Figure 4 might not be wrongly classified.

\subsection{Low Performance Increases in Reuters-21578}

Our algorithm was able to increase the categorization performance on Yahoo-Science significantly, but not as much on Reuters-21578. This seems to be because our algorithm is good at increasing recall but not as good at increasing precision. Table 6 shows the recall and precision rates before and after adding bigrams. 
Table 6. Recall and Precision without and with bigrams

\begin{tabular}{|c|c|c||c|c|}
\hline \multirow{2}{*}{ Corms } & \multicolumn{2}{|c||}{ Yahoo-Science } & \multicolumn{2}{c|}{ Reuters-21578 } \\
\cline { 2 - 4 } & Recall & Precision & Recall & Precision \\
\hline Unigrams only & $58.8 \%$ & $75.4 \%$ & $88.6 \%$ & $57.9 \%$ \\
\hline Unigrams + Bigrams & $65.1 \%$ & $76.7 \%$ & $90.8 \%$ & $57.6 \%$ \\
\hline
\end{tabular}

Apparently, our algorithm works by increasing recall more than precision. This is the way it is expected to work, as the algorithm uses bigrams to reinforce existing unigrams and most of the bigrams found by our algorithm are from the positive category. Hence, they work better on the positive documents than the negative ones. In other words, our algorithm is better at increasing correct positives than at reducing false positives. Hence, it works best in cases where recall is originally low because, in such cases, our algorithm can increase the performance by increasing correct positives.

The evidence we found from our experimental results agreed with our hypothesis. Table 7 shows how the documents were reclassified when bigrams were added. Additional correct positive/negative documents were those correctly classified as positive/negative due to the addition of bigrams. Additional false positive/negative documents were those wrongly classified as positive/negative due to the addition of bigrams. 
Table 7. Increases in correct/false positives/negatives upon adding bigrams

\begin{tabular}{|l|c|c|}
\hline & Yahoo-Science & Reuters -21578 \\
\hline Correct positives & 686 & 82 \\
\hline Correct negatives & 182 & 156 \\
\hline False positives & 237 & 222 \\
\hline False negatives & 52 & 19 \\
\hline Net increase in correct positives & 634 & 63 \\
\hline Net increase in correct negatives & -55 & -66 \\
\hline
\end{tabular}

The number of additional positive documents correctly classified (Increase in correct positives - Increase in false negatives) increased in both Yahoo-Science and Reuters21578. But the number of additional negative documents correctly classified (Increase in correct negatives - Increase in false positives) decreased in both cases. This suggested that the algorithm was much more successful in increasing correct positives than correct negatives. That was why it performed much better on Yahoo-Science, where the recall rate was originally low, than in Reuters-21578, where recall was already very high to start with.

\subsection{The Main Strength and Weakness of the Algorithm}

As demonstrated in the preceding subsection, the strength of our algorithm is its ability to increase the number of positive documents classified correctly, but its weakness is that it may cause more negative documents to be classified incorrectly. 
The most likely reason is that our algorithm favors finding bigrams from the positive category. Indeed, we found that of all the bigrams found in our experiments, less than $5 \%$ came from the negative category. This happened because we used the same criteria for finding bigrams in both categories, but the size of the positive category tended to be much smaller than that of negative category.

\section{Conclusion}

In this paper, we proposed an efficient algorithm to enhance the performance of text categorization using bigrams. The algorithm was tested on two different corpora, yielding improved performances. However, the algorithm behaved poorly on some categories; we did some analysis to pinpoint the reason.

We found that the algorithm caused more negative documents to be classified incorrectly.

One way to remedy the problem is to find more bigrams from the negative category by using different parameters. Another idea is to combine the bigrams we found in other categories and use them in the negative category. Yet another approach is a two-stage classifier. The first stage basically consists of the classifier we currently have. If the document is classified as positive in the first pass, we run it through the second stage. In this stage, we classify the document again using a different set of classifier weights. The idea behind the second stage is to increase the precision by filtering out negative documents that are very close to positive documents 
The corpora we used for the current study might have contributed to the poor performances in some categories. It would be desirable to run the experiment on the other standard corpora as well. This is the research direction we will follow in the near future.

\section{Acknowledgement}

Part of the research was supported by NSF grant IIS-9908441. Taejon University supported the third author for his sabbatical year. He is grateful for providing him with computing facilities to the Department of Computer Science, University of California, Santa Barbara where this work was performed.

\section{References}

Apté, C., Damerau, F., and Weiss, S. (1994). Automated learning of decision rules for text categorization. ACM Transactions on Information Systems, 12(3), 233-251.

Apté, C., Damerau, F., and Weiss, S. (1998). Text Mining with Decision Trees and Decision Rules. Presented at the Conference on Automated Learning and Discovery. Pittsburgh, PA.

Billsus, D. and Pazzani, M. (1997). Learning Probabilistic User Models. In Workshop Notes of "Machine Learning for User Modeling," Sixth International Conference on User Modeling, Chia Laguna, Sardinia. 
Dietterich, T. (1997). Approximate Statistical Tests for Comparing Supervised Classification Learning Algorithms. Neural Computation, 10(7), 1895-1924.

Fürnkranz (1998). A Study Using ngram features for Text Categorization. Technical Report OEFAI-TR-98-30, Austrian Research Institute for Artificial Intelligence, Vienna, Austria.

Lewis, D. (1992a). Representation and Learning in Information Retrieval. Technical Report UMCS-1991-093. Department of Computer Science, University of Massachusetts, Amherst, MA.

Lewis, D. (1992b). Feature selection and feature extraction for text categorization. In Proceedings of a Workshop on Speech and Natural Language, (pp. 212-217). San Mateo, CA: Morgan Kaufmann.

Lewis, D. (1992c). An evaluation of phrasal and clustered representations on a text categorization task. In Croft et. al. (Ed.), Proceedings of SIGIR-95, $15^{\text {th }}$ ACM International Conference on Research and Development in Information Retrieval (pp. 37-50). New York: ACM Press

Lewis, D. (1992d). Text representation for intelligent text retrieval: A categorization-oriented view. In Jacobs, P. (Ed.), Text-Based Intelligent Systems, (pp. 179-197). Hillsdale, NJ: Lawrence Erlbaum.

Lewis, D. and Ringuette, M. (1994). A comparison of two learning algorithms for text categorization. In Third Annual Symposium on Document Analysis and Information Retrieval, (pp. 81-93). Las Vegas, NV. 
Lewis, D. (1998). Naive (bayes) at forty: The independence assumption in information retrieval. In Proceedings of ECML-98, $10^{\text {th }}$ European Conference on Machine Learning, (pp. 415). Chemnitz, Denmark.

McCallum, A. (1996). Bow: A toolkit for statistical language modeling, text retrieval, classification and clustering. http://www.cs.cmu.edu/ mccallum/bow.

McCallum, A. and Nigam K. (1998). A Comparison of Event Models for Naive Bayes Text Categorization. Presented to AAAI-98 Workshop on Learning for Text Categorization.

Mladeni(D), D. and Grobelnik, M. (1998). Word sequences as features in text learning. In Proceedings of the $17^{\text {th }}$ Electrotechnical and Computer Science Conference (ERK-98) (pp. 145148), Ljubljana, Slovenia.

Salton, G. and McGill, M. (1983). Introduction to Modern Information Retrieval. New York: McGraw-Hill.

Schapire, R, Singer, Y., and Singhal, A. (1998). Boosting and Rocchio Applied to Text Filtering. In Croft et. al. (Ed.), Proceedings of SIGIR-98, $21^{\text {st }}$ ACM International Conference on Research and Development in Information Retrieval (pp. 215-223). New York: ACM Press.

Schütze, H., Hull, D., and Pederson, J. (1995). A Comparison of Classifiers and Document Representations for the Routing Problem. In Croft et. al. (Ed.), Proceedings of SIGIR-95, $15^{\text {th }}$ ACM International Conference on Research and Development in Information Retrieval (pp. 229237). New York: ACM Press 
Sebastiani, F. (1999). Machine Learning in Automated Text Categorisation: A Survey. Technical Report IEI-B4-31-1999, Instituto di Elaborazione dell'Informazione, Consiglio Nazionale delle Richerche. Pisa, Italy.

Tan, C.-M. (2000). Finding and Using High Quality Word-Pairs for Enhanced Text Categorization. Unpublished Master's Thesis. Department of Computer Science, University of California, Santa Barbara.

Yang, Y. and Pedersen J. (1997). A Comparative Study on Feature Selection in Text Categorization In Proceedings of ICML-97, $14^{\text {th }}$ International Conference on Machine Learning, (pp. 412-420). Nashville, TN.

Yang, Y. and Liu, X. (1999). A re-examination of text categorization methods. In Croft et. al. (Ed.), Proceedings of SIGIR-99, $22^{\text {nd }}$ ACM International Conference on Research and Development in Information Retrieval (pp. 42-49). New York: ACM Press. 